Western University Scholarship@Western

1984

\title{
A Rehabilitation of Absolute Advantage
}

Glenn M. MacDonald

James R. Markusen

Follow this and additional works at: https://ir.lib.uwo.ca/economicsresrpt

Part of the Economics Commons

Citation of this paper:

MacDonald, Glenn M., James R. Markusen. "A Rehabilitation of Absolute Advantage." Department of Economics Research Reports, 8410. London, ON: Department of Economics, University of Western Ontario (1984). 
ISBN : $\quad 0-7714-0544-8$

\title{
RESEARCH REPORT 8410
}

A REHABILITATION OF ABSOLUTE ADVANTAGE*

\author{
Glenn M. MacDonald \\ James R. Markusen
}


MacDonald, G. M. ; Markusen, J. R.

A Rehabilitation of Absolute Advantage

This paper considers the question of how optimal assign-

ments depend on the endowed traits of economic agents. Its

most useful point is that the absolute levels of these traits

always, in conjunction with technology, determine the structure

of efficient allocations. Only under very special circumstances

do summary measures of the structure of traits, such as compara-

tive advantage, succeed in performing this function unless they

do so tautologically.

Department of Economics

University of Western Ontario

London, Ontario N6A $5 \mathrm{C} 2$

Canada. 
Assignment problems form an important class of questions in a wide range of sub-disciplines of economic science. In labor economics the assignment of workers to tasks within the firm, or family members within a household, is studied; international trade theory has one of its principal concerns the assignment of countries to commodities; operations research analyzes problems such as the assignment of limited capacity to product lines, and so forth.

The Principle of Comparative Advantage has become something of an article of faith concerning the solutions to many of these assignment problems. While there does not appear to be any unambiguous definition, assignment by comparative advantage is usually taken to mean assignment on the basis of a summary measure of endowed traits, particularly relative charasteristics, whether they be workers' skills, national factor endowments, or other attributes. In a simple two-attribute case, an assignment by comparative advantage can therefore be defined as an assignment on the basis of the ratio of the two attributes possessed by the individual workers, country, etc.

It is clear that this was the original intention of Ricardo, who is widely credited with first establishing the Principle of Comparative Advantage. Ricardo argued that assignment of countries to the production of goods on the basis of relative labor productivities was optimal quite independently of absolute productivities. The Heckscher-Ohlin model of trade continued this tradition by showing that countries should be assigned to (though not necessarily fully specialized in) goods using intensively the country's relatively abundant factors. Once again, the optimal assignment depends only on relative attributes, in this case factor endowments. 
More recently, the Principle of Comparative Advantage has been forma1ly established in labor economics. Rosen (1978) analyzed optimal assignment of heterogeneous workers. For a particular type of technology, he obtains the following very strong result. Suppose there are just two activities $(\alpha$ and $\beta$ ) in which workers might engage, and many types of workers. Then, in an optimal assignment, each worker participates in only one activity. Further, when worker types are ordered according to declining comparative advantage at activity $\alpha$ relative to activity $\beta$, there is a marginal worker type such that all workers with comparative advantage greater than that of this marginal worker type performs only activity $\alpha$, the rest performing $\beta$.

Although these results present a strong case, assignment by comparative advantage does not square well with experience. It is not persuasive that the employee with the highest comparative advantage in management should become president. Indeed it is plausible that the presidency assignment will have something to do with absolute advantage; alternatively, a person with poor management skills will not be chosen even if he is relatively worse at every other task in the firm. Further, in academic economics, generally poor economists will not be chosen as department chairmen even : if they have a comparative advantage in these activities relative to research and teaching.

International trade economists have recently been coming to grips with these issues. A prediction that follows from the Ricardian and HeckscherOhlin comparative-advantage models is that identical economies will not trade. Yet, that countries with apparently similar technologies and factor endowments seem to trade large volumes of manufactured goods with one another can 
be taken as evidence that assignments do not depend entirely on comparative advantage. Indeed, trade arising due to scale economies in models with identical countries is now referred to as "non-comparative-advantage trade" (Melvin 1969, Krugman 1979). That specialization may be optimal independently of differences in comparative advantage is a special case of a more general failure of the comparative advantage principle to predict as signments.

The purpose of this paper is to construct a model with a fairly general technology and examine the structure of optimal assignment based upon it. The assignment of workers within a firm is taken to be the motivating example, with applications to other assignment problems considered toward the end of the paper. In the firm model, heterogeneous workers must divide their time (they need not specialize) between two tasks, $\alpha$ and $\beta$. Among other things, it is shown that only in a razor's edge case can comparative advantage in terms of workers' relative talents in performing the tasks required for production predict the structure of the optimal assignment. In contrast, absolute skill levels along with technology, determine this structure. It is in this sense that absolute advantage is rehabilitated by this analysis.

The essential features of the technology used to reach this conclusion are that: (i) workers of a given skill level may experience increasing or decreasing marginal product with respect to the amount of one task performed; and (ii) one task may be a public input such as research or administrative within the firm. Decreasing marginal product arises for the usual variable proportions reasons, a concept which could be interpreted as covering boredom and fatigue. Increasing marginal product corresponds to a warmup notion or indivisibilities within the task itself. The existence of a public or joint 
input is a justification for the existence of the firm in the first place, and implies that workers performing this task create an external effect on the output of other workers.

The paper is structured as follows. Section 1 describes the labor force and workers' endowed traits, the maximization problem faced by firms making up Section 2. Optimal assignments are characterized in Section 3, with extensions to other settings following in Section 4. The final section summarizes and concludes.

1. SKILIS AND THE IABOR FORCE

Each worker in the labor force is endowed with the ability to perform two tasks, called $\alpha$ and $\beta$, where only one task may be undertaken at any point in time. There are many different types of workers, differentiated by the quantity of tasks $\alpha$ and $\beta$ which each can execute in one period of work; $t \equiv\left(t_{\alpha}, t_{\beta}\right)$ is the vector of such attributes. Thus a worker of type $t$ can perform $t_{\alpha}$ of task $\alpha$, or $t_{\beta}$ of task $\beta$, or some combination depending upon the allocation of time over tasks.

The supply side of the labor market is completely described by a continuous density $L(\cdot)$, having support $\Omega \subset R_{+}^{2}$. This specification assumes the period of work is predetermined and that workers regard both tasks as equally distasteful.

2. THE FIRM'S PROBLEM

In this section the maximization problem faced by firms is set out. Initially, attention is focused on the technology available to firms. Subsequently it is shown that the optimal assignment problem can be studied as a particularly straightforward first stage in a two-stage maximization problem. 
Firms are assumed to be perfect competitors in both product and factor markets, selling output $\mathrm{X}$ at price $\mathrm{p}$. All have access to the same technology, in which labor is the only variable factor of production. The number of workers of type $t$ employed by the firm is described by a measurable function $\ell(t)$, positive for $t \in \omega$, where $w \subset \Omega$ is chosen by the firm. The wage paid to workers of type $t$ is $w(t)$, where $w(\cdot)$ is positive, monotonically increasing in both arguments, and continuous. The wage depends only on a worker's skills (and specifically not on his assignment) by virtue of the assumption that both tasks are equally distasteful.

The technology available to the firm delineates the relationship among output, the number of workers of each type, the skills of each, and the manner in which the workers' time is allocated between tasks. Output (X) is a monotonically increasing and strictly concave function of the aggregate (Q) of individual outputs $(q(\cdot))$ :

$$
\mathrm{x}=\mathrm{x}(\mathrm{Q}), \mathrm{x}^{\prime}>0, \mathrm{x}^{\prime \prime}<0 \text { 。 }
$$

$X(\cdot)$ is concave as a result of the existence of fixed factors, ignored in what follows. $Q$ is assumed to be a linear aggregate of individual outputs $q$ :

$$
Q=\int_{\omega} q\left[\varphi(t), t_{\alpha}, \xi(t) T_{\beta}\right] \ell(t) d t
$$

The individual output of a single worker of type $t(q(t))$ requires (i.e., all inputs are necessary) inputs of time spent on task $\alpha(\varphi(\bullet))$, skill at task $\alpha$ $\left(t_{\alpha}\right)$, and an allocation $\left(\xi(\cdot) T_{\beta}\right)$ of the aggregate quantity of task $\beta$ performed $\left(T_{\beta}\right) 0^{1}$ The function $\xi(0)$ describes the allocation of $T_{\beta}$ across workers, to be explained shortly。 Note from (2) that in general one worker's time spent performing task $\alpha$ does not substitute perfectly for that of another in the production of final output. Imperfect substitutability occurs because at the individual level, the marginal product of time spent performing task $\alpha$ is not 
necessarily constant. Indeed, the notion that the marginal product of time spent performing task $\alpha$ may be diminishing for the usual variable proportions reasons--rising ratio of $\varphi$ to $t_{\alpha}$ and $\xi(\cdot) \mathrm{T}_{\beta}{ }^{--}$or increasing as a result of indivisibilities, is key to the discussion of specialization, or the lack thereof, contained in the sections to follow.

The total quantity of task $\beta$ is given by

$$
T_{\beta}=\int_{\omega}[1-\varphi(t)] t_{\beta} \ell(t) d t
$$

Equation (3) implies there is perfect substitutability (in the production of aggregate task $\beta$ ) between workers' time spent performing task $\beta$. Perfect substitutability is not crucial for the general flavor of the results presented below, but this specification permits an especially simple characterization of the optimal assignment.

The production of shirts provides an illustration of the ideas behind the structure (1) - (3)。 Task $\alpha$ is the actual sewing of cloth into shirts, and task $\beta$ the production of cloth, as well as administration or pattern design. Each worker is allocated a portion of the latter collection which he combines with his time and talent at sewing, to construct shirts (q). Shirts are aggregated to arrive at the total production of shirts (Q), which are then transported and distributed using fixed inputs (not modelled) to arrive at "marketed shirts", $\mathrm{X}(\mathrm{Q})$ 。

The function $\xi(t)$ delineates the manner in which $T_{\beta}$ is shared out among workers. $\xi(t) \geq 0$ satisfies

$$
\int_{\omega} \xi(t) l(t) d t=f(<b)
$$

$f(<b)$ is a positive, real valued, functional defined on measurable functions $l(\cdot)$ representing the firm's labor force. The basic notion is that 
the way in which $T_{\beta}$ may be. shared out can depend on both the size and composition of the firm's workforce. A number of special cases are of interest. $f(\cdot) \equiv 1$ implies $T_{\beta}$ is a purely private input, of which eash worker of type $t$ receives a share $\xi(t)$. For example, task $\beta$ could be the cutting of material in the shirt factory. A special case of this purely private input setup is that in which $\xi(t) T_{\beta}=[1-\varphi(t)] t_{\beta}$; that is, each worker does all his own cutting, implying individual production functions are of the form $q\left[\varphi, t_{\alpha},(1-\varphi) t_{\beta}\right]$.

In contrast, $f(\cdot)=\int$ edt allows $T_{\beta}$ to be treated as a pure public input $(\xi(\cdot) \equiv 1)$, examples of which are administrative activities providing a work environment within which task $\alpha$ is undertaken, or a design for a shirt. Blueprints and related concepts of technology, knowledge, and information are all examples of public inputs. Once a design is developed, it can be given to any number of additional workers without reducing the value of the design to other workers. More generally, for workforces $\ell^{1}$ and $\ell^{2}$ which are distinct but of equal size (i.e., $\left.\int l^{1} \mathrm{dt}=\int l^{2} \mathrm{~d} t\right), f\left(\left\langle l^{1}\right\rangle\right) \neq f\left(\left\langle l^{2}\right\rangle\right.$ ) embodies the idea that the private/public nature of the shared input $T_{\beta}$ depends on the composition of the workforce. For example, $\left.\left.f\left(<l^{1}\right\rangle\right)>f\left(<l^{2}\right\rangle\right)$ can be interpreted as $\ell^{1}$ requiring less supervision than $\ell^{2}$. In any case, so long as $f(\cdot)>1$ there is an element of jointness, providing a reason for some collection of workers to work together.

The qualitative characteristics of the assignments studied below do not depend in any important way on the extent to which $T_{\beta}$ is public or private, or whether $\xi(\cdot)$ is a matter of choice given its public/private characteristics. Since much algebra is avoided in the process, $T_{\beta}$ will be treated as a pure public $\operatorname{good}: \quad \xi(\cdot) \equiv 1^{2}$ 
Given the technology available to the firm, the profit maximization problem is

$$
\begin{aligned}
& \underset{\varphi(t), \ell(t)}{\max } \operatorname{px}\left\{\int_{\omega} q\left[\varphi(t), t_{\alpha}, T_{\beta}\right] \ell(t) d t\right\}-\int_{\omega} w(t) l(t) d t-F \\
& \text { S.T. (i) } \forall t e w, 0 \leq \varphi(t) \leq 1 \text { and } \ell(t) \geq 0 ; \\
& \text { and (ii) } T_{\beta}=\int_{\omega}[1-\varphi(t)] t_{\beta} l(t) d t ;
\end{aligned}
$$

where $\omega=\{t \mid \ell(t)>0\}$ is the support of $\ell(t)$ and $F$ is the cost of fixed factors. This problem is greatly simplified by noting the following. Recall that under the assumption that individuals find all tasks equally distasteful, $w(\cdot)$ does not depend on $\varphi(\cdot)$. Given this result, the full profit-maximizing problem can be viewed as choosing $\varphi(\cdot)$ to maximize $Q$ for fixed $\ell(\bullet)--$ the "assignment problem", yielding a maximal Q given $\ell(\cdot)$ denoted by $\Psi(\langle\ell(t)\rangle)$, the "derived" production functional--then choosing $\ell(0)$ with $\varphi\left(0^{\circ}\right)$ varying optimally - the standard factor proportions problem. 3

The assignment problem can be studied in isolation provided the assumed $\ell(\cdot)$ is of the form which will emerge in equilibrium. The analysis to follow assumes $w$ does not consist of isolated points (i.e., firms do not specialize in hiring a comparatively small number of types of workers). This assumption is consistent with equilibrium outcomes provided $\Psi\left(0_{0}\right)$ is a strictly concave functional. In this case, factor demands are constructed from $\Psi(\cdot)$ in the usual fashion, and market equilibrium can be constructed as follows. Aggregation over firms yields total factor demand $D(\langle w(t)\rangle)$. Supply is $L(\cdot)$, discussed above. Assuming the optimal firm size is not too large, free entry will (recall $\mathrm{F}>0$ ) yield an equilibrium in which wages are such that firms choose $\ell(\cdot)=L(\cdot) / N$, where $N$ is the equilibrium number of firms, and $\omega=\Omega_{0}$ Thus, assuming concavity of $\Psi$, the assignment problem can be studied in isolation for arbitrary $\ell(\cdot)$ which are continuous on $\Omega .^{4}$ 
Proceeding in this manner, the individual firm's assignment subproblem is

$$
\varphi(t) \epsilon[0,1] \quad \max _{\Omega} q\left\{\varphi(t), t_{\alpha}, \int_{\Omega}[1-\varphi(\nu)] \nu_{2} l(\nu) d \nu\right\} \ell(t) d t
$$

where $\nu=\left(\nu_{1}, \nu_{2}\right)$ is a vector integration dummy ranging over $\Omega$.

First-order necessary conditions for a maximum are (letting $\varphi^{*}(t)$

denote the optimal values)

$$
q_{1}(\cdot)-t_{\beta} \mu= \begin{cases}z 0 & \text { if } \varphi *(t)=1 \\ =0 & \text { if } 0<\varphi^{*}(t)<1 \\ \leqslant 0 & \text { if } \varphi *(t)=0,\end{cases}
$$

where $\mu \equiv \partial Q / \partial T_{\beta}=\int q_{3}(\cdot) \ell(t) d t>0 . \mu$ is the efficiency price of a unit of task $\beta ; q_{1}$ and $t_{\beta} \mu$ are the marginal products (in terms of $Q$ ) of time spent on tasks $\alpha$ and $\beta$, respectively. Note that $\mu$ does not vary across worker types, which is the simplification obtained by assuming $T_{\beta}$ to be a pure public good.

Optimal time allocations $\varphi *(\cdot)$ can take on numerous forms. The two of primary interest are those for which (i) $0<\varphi^{*}(t)<1$ for each t--all workers are "diversified"; and (ii) $\varphi *(t)=0$ or 1 for each $t--a l l$ workers are specialized. A condition necessary for pure diversification is that for all worker types $t$,

$$
\frac{\partial^{2} Q}{\partial \varphi(t)^{2}}=q_{11}(\cdot)-2 q_{13}(\cdot) t_{\beta} l(t)+t_{\beta}^{2} l(t) \int_{\Omega} q_{33} l(\nu) d \nu<0,
$$

where the left-hand side is evaluated for $\varphi^{*}(t)$ satisfying (5) as an equality. (6) could fail due to increasing marginal product of either $\varphi$ or $T_{B}$ in individual production, in which case specialization is implied. 


\section{OPTIMAL ASSIGNMENTS}

In this section, the solution to the optimal assignment problem is characterized. For the sake of brevity, attention is confined to the purely diversified and specialized cases, which are pursued in that order. The manner in which time allocation, individual output and wage rates vary across worker types is readily established. Following that, the question of whether optimal assignments can be characterized in terms of the pattern of comparative advantage is examined. In order for this idea to have any content, there must be clear definitions of comparative advantage, an assignment, and what it means for comparative advantage to dictate that assignment. The definitions employed here are as follows. Relative to a worker of type $t^{1}$, a worker of type $t^{0}$ has a comparative advantage at task $\alpha$ if and only if $t_{\alpha}^{0} / t_{\beta}^{0}>t_{\alpha}^{1} / t_{\beta^{0}}^{1}$ Assignment refers to time allocated to task $\alpha, \varphi(\cdot)$. Finally, an assignment $\varphi(\cdot)$ is said to follow the pattern of comparative advantage if and only if whenever two worker types are compared, the type having a comparative advantage at task $\alpha$ is optimally allocated more time performing task $\alpha_{0}$

Given these definitions, it is demonstrated that, except for a razor's edge case, for each worker type $t$ there are worker types having comparative advantage (disadvantage) at task $\alpha$ who are assigned less (more) time performing that task. 5

This is a strong result, and the reader may wish to quarrel with the definition of comparative advantage and assignment on which it is based. Yet the arguments applied below appear to be effective whenever comparative advantage and assignment are not defined so as to be identical. In brief, only under the rarest of circumstances is it possible to picedict the pattern 
of assignment given only summary information on the structure of skills. Some relevant information about skills and their interaction with technology is almost invariably lost. $^{6}$

Optimal Diversification

An optimal diversified time allocation $\varphi^{*}(t)$ solves

$$
\mathrm{q}_{1}\left[\varphi^{*}(t), \mathrm{t}_{\alpha}, \mathrm{T}_{\beta}\right]-\mathrm{t}_{\beta} \mu=0 \quad \forall t \in \Omega
$$

$\varphi^{*}(t)$ simply equates the marginal value of time in each activity.

The manner in which $\varphi^{*}(t)$ varies across individuals is obtained from differentiation of (7) with respect to elements of $t$ :

and

$$
\frac{\partial \varphi^{*}}{\partial t_{\alpha}}=\frac{q_{12}}{-q_{11}} \geq 0,
$$

$$
\frac{\partial \varphi^{*}}{\partial t_{\beta}}=\frac{\mu}{q_{11}}<0 .
$$

Note that since the experiment involves comparing individual workers with different abilities, as opposed to changing the skills of a given worker, $\mu$ and $T_{\beta}$ remain constant. Also, though not strictly required by the second-order conditions, $\mathrm{q}_{11}<0$ is assumed.

Workers having greater facility in the performance of task $\alpha$ will spend more or less time performing that task according to whether greater ability raises or lowers the marginal product of time; $q_{12}>0$ will be taken to be the leading case. But those who are more able at task $\beta$ always spend less time performing task $\alpha$. This occurs because all workers utilize the same quantity of task $\beta$ in individual production. Raising $t_{\beta}$ thus merely increases the opportunity cost of time spent on task $\alpha$.

Equations (9) and (10) permit construction of $\varphi$-constant loci in ת. One such locus is depicted in Figure 1 , and labelled $\bar{\varphi}$. In the 
figure, the arrow emanating from $\bar{\varphi}$ represents the direction in which loci corresponding to greater $\varphi^{*}(t)$ lie. The slope of the $\varphi$-constant locus is

$$
\left.\frac{d t_{\beta}}{d t}\right|_{d \varphi^{*}=0}=q_{12} / \mu \text {. }
$$

The pattern of individual output across workers may also be obtained. Since individual output is $q\left[\varphi^{*}(t), t_{\alpha}, T_{\beta}\right]$ and $T_{\beta}$ does not vary across workers, and

$$
\frac{\mathrm{dq}}{\mathrm{dt}{ }_{\alpha}}=\mathrm{q}_{1} \frac{\partial \varphi^{*}}{\partial \mathrm{t}_{\alpha}}+\mathrm{q}_{2} \stackrel{\gtrless}{<},
$$

$$
\frac{d q}{d t_{\beta}}=q_{1} \frac{\partial \varphi^{*}}{\partial t_{\beta}}<0
$$

Workers who are more able at task $\alpha$ will produce more output simply because $t_{\alpha}$ is an input, and less to the extent that they might be allocated less time (if $\mathrm{q}_{12}<0$ ). Using (8), it can be shown that dq/dt $\alpha<$ if and only if $\frac{\partial}{\partial \varphi}\left(-q_{1} / q_{2}\right)<0$, which is equivalent to $t_{\alpha}$ being an inferior factor of production holding $\mathrm{T}_{\beta}$ constant. ${ }^{7}$ Thus $\mathrm{dq} / \mathrm{dt} \mathrm{t}_{\alpha}>0$ is to be expected. On the other hand, since $t_{\beta}$ does not enter individual production directly, and Increments to $t_{\beta}$ reduce $\varphi^{*}$, workers who are more able at performing task $\beta$ always produce less output.

$q$-constant loci are represented in Figure 1 and labelled $\bar{q}$. Using (8) $-(11)$ it may be checked that for any $t$, the $q$-constant: locus through that point is always steeper than the corresponding $\varphi$-constant locus :

$$
\left.\frac{d t_{\beta}}{d t_{\alpha}}\right|_{d q=0}=\frac{q_{12}}{\mu}-\frac{q_{2}}{q_{1} \partial \varphi^{*} / \partial t_{\beta}}>\left.\frac{d t_{\beta}}{d t_{\alpha}}\right|_{d \varphi^{*}=0} .
$$

The argument is streightforward. An increment to $t_{\alpha}$ requires some adjustment (of the same sign as $q_{12}$ ) to $t_{\beta}$ if $\varphi$ is to be held fixed. But given this adjustment, $q$ must be above its initial value because $t_{\alpha}$ is greater and both $\varphi$ and $T_{\beta}$ are unchanged. Thus $t_{\beta}$ must be raised still further to reduce $\varphi$ sufficiently to return $q$ to its original value. 
The impact of changes in $t$ on wage rates is obtained as follows. The value of the marginal product of workers of type $t$ is $\mathrm{px}^{\prime}(\cdot)\left\{\mathrm{q}(\cdot)+\left[1-\varphi^{*}(t)\right] \mathrm{t}_{\beta}{ }^{\mu}\right\}$, which will also be their equilibrium wage rate $w(t)$. Using the envelope theorem, worker characteristics are valued at rates

$$
\frac{\partial w}{\partial t_{\alpha}}=p x^{\prime}(\cdot) q_{2}
$$

and

$$
\frac{\partial w}{\partial t_{\beta}}=p x^{\prime}(\cdot)\left[1-\varphi^{*}(t)\right] \mu .
$$

Of particular interest are comparisons of workers whose skills differ only by scale. For any worker of type $t$, workers whose skills are the same up to scale have abilities $\zeta t$, where $\zeta$ is a scalar equal to unity for the "base" worker. In Figure 1, such workers are arranged along the ray from the origin; workers of type $\underline{t}$ are the "base".

Differentiation of (7) gives

$$
\frac{d \varphi^{*}}{d \zeta} \div \eta-1
$$

where $\eta \equiv t_{\alpha} q_{12} / q_{1}$ is the elasticity of the marginal product of $\varphi$ with respect to $t_{\alpha^{*}}$ Raising $\zeta$ increases the value of time in both activities. Whether $\eta$ exceeds or falls short of unity determines whether the marginal value of time spent performing task $\alpha$ rises faster or slower than the value of time on task $\beta$. Though $\eta>1$ can hold even for concave $q(0)$ and $q_{12}<0$ implies $\eta<0,0<\eta \leq 1$ is evidently the leading case. Thus workers who are proportionately more able will generally spend less time performing task $\alpha: \mathrm{d} \varphi^{*} / \mathrm{d} \zeta<0$. Whether workers who are proportionately more able produce more output is less clear : 


$$
\frac{\mathrm{dq}}{\mathrm{d} \zeta}=\mathrm{q}_{1} \frac{\mathrm{d} \varphi^{*}}{\mathrm{~d} \zeta}+\mathrm{q}_{2} \mathrm{t}_{\alpha} \stackrel{\gtrless}{<}
$$

though, of course, such workers must earn more:

$$
\frac{d w}{d \zeta}=q_{2} t_{\alpha}+\left[1-\varphi^{*}(t)\right]_{\mu} t_{\beta}>0
$$

Further, whether earnings rise proportionately more or less than the increase in skills depends on whether $q(\cdot)$ is concave or convex in $t_{\alpha}$ :

$$
\left.\frac{1}{w} \frac{d w}{d \zeta}\right|_{\zeta=1}=\frac{q_{2} t_{\alpha}+\left[1-\varphi^{*}(t)\right]_{\mu t}}{q+\left[1-\varphi^{*}(t)\right] \mu t_{\beta}} \gtreqless 1 \Leftrightarrow q_{2} t_{u} \gtreqless q .
$$

Now, does comparative advantage determine the pattern of assignment?

Recall that one assignment follows the pattern of comparative advantage if and only if workers who have a comparative advantage at task $\alpha$ are allocated more time performing that task.

A condition necessary and sufficient for $\varphi^{\circ}(t)$ to follow the pattern of comparative advantage is $\eta=1$ when $q(\cdot)$ is evaluated at $\left(\varphi^{t}(t), t_{\alpha}, T_{\beta}\right)$. If and only if this condition holds do all workers having a given $t_{\alpha} / t_{\beta}$ allocate their time the same way (equation (12)). Moreover, higher $t_{\alpha} / t_{\beta}$ yields increased $\varphi^{*}(t)\left(\eta=1\right.$ implies $q_{12}>0$ in $\left.(8)\right)$.

The sufficiency part of the argument is obvious from (12). Necessity provides a 1ittle extra information. If $\varphi^{*}(t)$ does not vary with proportional changes in $t, \eta=1$ is implied, which can be regarded as a differential equation in $q_{1}(\cdot):^{8}$

$$
t_{\alpha 12} q_{1}\left(\varphi^{*}, t_{\alpha}, T_{\beta}\right)-q_{1}\left(\varphi^{*}, t_{\alpha}, T_{\beta}\right)=0
$$

The solution is of the form

$$
q_{1}\left(\varphi, t_{\alpha}, T_{\beta}\right)=q^{o}\left(\varphi, T_{\beta}\right) t_{\alpha}
$$

for some function $q^{\circ}(\cdot)$. Integration over $\varphi$ recovers the technology

$$
q\left(\varphi, t_{\alpha}, T_{\beta}\right)=q^{1}\left(\varphi, T_{\beta}\right) t_{\alpha}+q^{2}\left(t_{\alpha}, T_{\dot{\beta}}\right)
$$


for some $q^{1}(\cdot)$ and $q^{2}(\cdot)$. That all factors are necessary implies $q^{2}(\cdot) \equiv 0$. For this technology (7) can be rewritten

$$
\frac{q^{0}\left(\varphi, T_{\beta}\right)}{. \mu}=\frac{t_{\beta}}{t_{\alpha}}
$$

in which case, the dependence of $\varphi$ on $t_{\beta} / t_{\alpha}$ only is immediate and arises because marginal returns and costs are proportional to $t_{\alpha}$ and $t_{\beta}$ respectively.

The lack of correspondence between optimal assignment and assignment by comparative advantage can be restated as follows. For worker type $t$, and associated time allocation $\varphi^{*}(t)$, if $\eta \neq 1$ there exist both: (i) workers who have a comparative advantage at task $\alpha$ and spend less time performing that task; and (ii) workers who have a comparative disadvantage at task $\alpha$ and spend more time performing it. Referring to Figure 2, drawn from $0<\eta<1$, relative to workers of type $t^{\circ}$, worker types in the north-east (south-west) shaded area have a comparative advantage (disadvantage) at task $\alpha$ but spend less (more) time performing that task. The cases $\eta \leq 0$ and $\eta>1$ can be treated in a similar fashion.

Since assignment by comparative advantage holds if and only if $q\left(\varphi, t_{\alpha}, T_{\beta}\right)=q^{o}\left(\varphi, T_{\beta}\right) t_{\alpha}$, it is worth enquiring into whether this is a strong restriction. First of all, for any $q\left(\varphi, t_{\alpha}, T_{\beta}\right)$ is it possible to redefine units of $t_{\alpha}$ so that $q(\cdot)$ is linear in the new units of $t_{\alpha}$ ? That is, does there exist a function $\hat{t}\left(t_{\alpha}\right)$ with $\hat{t}^{\prime}>0$, such that $q\left(\varphi, t_{\alpha}, T_{\beta}\right)=\hat{q}\left(\varphi, T_{\beta}\right) \hat{t}_{\alpha}$ If this were so, appropriate choice of units would always yield comparative advantage determining assignment. Blackorby et al. (1978) show that such a change of units is possible if and only if $q(\cdot)$ has the property $\partial \log q / \partial \varphi=\bar{q}\left(\varphi, T_{\beta}\right)$ for some $\bar{q}(\cdot)$ not depending on $t_{\alpha}$; equivalently, $q=\hat{q}\left(\varphi, T_{\beta}\right) h\left(t_{\alpha}\right) \cdot q(\cdot)$ must be multiplicatively separable. That multiplicative separability is a strong restriction is easily established. Briefly, let 
$S$ be the set of production functions $q(\cdot)$, and $s^{M}$ be the subset of $S$ which is multiplicatively separable. Then it is easy to check that the complement of $s^{M}$ in $S$ (i.e., the set of production functions which are not multiplicatively separable) is open and dense in S. Roughly speaking, almost everything in $S$ is not multiplicatively separable. 9

All this is not to say that comparative advantage is completely irrelevant. Indeed, it is obvious that it is always possible to find two worker types whose optimal assignments agree with assignment by comparative advantage. In fact, recalling (8) and (9), for $\mathrm{q}_{12}>0$ changes in comparative advantage induced purely by increments to just one of $t_{\alpha}$ or $t_{\beta}$ generate adjustments which are always in accord with comparative advantage. This result obtains simply because changes in one of $t_{\alpha}$ or $t_{\beta}$ induce relatively large changes in $t_{\alpha} / t_{\beta}$. The difficulty arises when both $t_{\alpha}$ and $t_{\beta}$ vary. To see this point more clearly, write the equality of marginal products of time across activities as

$$
\left(\frac{t_{\alpha}}{t_{\beta}}\right)\left(\frac{q_{1}}{t_{\alpha}}\right)=\mu
$$

Holding $\mathrm{q}_{1} / \mathrm{t}_{\alpha}$ fixed, an increase in $t_{\alpha} / \mathrm{t}_{\beta}$ raises the left-hand side of (13). This effect, which may be called the comparative advantage effect (CA) always works in favor of increasing $\varphi_{0}$ But for given $t_{\alpha} / t_{\beta}$, an increase in $t_{\alpha}$ either raises, leaves constant, or lowers the factor $q_{1} / t_{\alpha}$ depending on whether $\eta$ exceeds, equals or falls short of unity. This effect, which can be labelled the absolute advantage effect (AA), can thus imply either greater or smaller $\varphi$ depending on the size of $\eta$ relative to unity and the direction in which $t_{\alpha}$ changes. Therefore, whether a given comparison of two worker types generates differences in assignments which are in accord with assignment by comparative advantage depends on the relative size of the $C A$ and $A A$ 
effects. For example, comparing worker types $t^{0}$ and $t^{1}$ in Figure 2, optimal assignments correspond to assignments by comparative advantage. However, for workers of type $t^{2}$ (having the same comparative advantage as $t^{1}$, but who are more able), relative to the comparison of types $t^{0}$ and $t^{1}$, the comparison of $t^{0}$ to $t^{2}$ has an identical CA effect but a much stronger AA effect owing to the larger change in $t_{\alpha}$. Consequently the AA effect dominates and the comparison of types $t^{0}$ and $t^{2}$ yields disagreement with assignment by comparative advantage. Loosely speaking, this larger AA effect is the reason the shaded region "fans out" to the right and left. That is, the $\varphi$-constant curve is the locus of vectors $t$ for which the AA and CA effects exactly cancel. When the AA effect is larger, a more substantial CA effect is required to offset it. Overa11, comparative advantage will tend to be a poor proxy for optimal assignment when differences in absolute advantage are large relative to differences in comparative advantage. 10

\section{Optimal Specialization}

When the optimal assignment involves pure specialization, the situation is similar to, but a good deal more straightforward than, the diversified case, The discussion is therefore brief.

For all workers of type $t$, either $\varphi^{*}(t)=1$ or $\varphi^{*}(t)=0$, depending on the sign of

$$
q\left(1, t_{\alpha}, T_{\beta}\right)-t_{\beta} \mu,
$$

where $\mu$, taking into account that $\varphi^{*}(t) \neq 0$ implies $\varphi^{*}(t)=1$, is as above. $q(\cdot)$ is the individual output of a worker of type $t$ who spends all of his time performing task $\alpha$. The second term is the value of the gain in the additional output of other workers occasioned by the type $t$ worker performing only task $\beta$, generating the public input.

Worker types $t$ for which the firm is indifferent about whether they perform task $\alpha$ or $\beta$ are those for which

$$
q\left(1, t_{\alpha}, T_{\beta}\right)-t_{\beta} \mu=0 .
$$


The set of such worker types is labelled $\phi$ in Figure 3 . The slope of $\tilde{\varphi}$ is $q_{2} / \mu>0$. The curvature of $\phi$ depends only on $q_{22}$. Figure 3 is drawn for $\mathrm{q}_{22}<0$.

Since $q_{2}>0$ and $\mu>0$, all workers to the south of $\tilde{\varphi}$ perform only task $\alpha$, the rest being assigned to $\beta$.

Turning to individual output, only those assigned to task $\alpha$ produce positive output. Among that group, those who are more able at task $\alpha$ produce more, while ability at task $\beta$ has no influence on output because it has no influence on time allocation. q-constant loci are vertical lines in $w$, with loci corresponding to greater output lying to the right. Wages are simply the marginal value of either own output or task $\beta$ : $p x^{\prime} q$ or $p^{\prime}{ }^{\prime} \beta^{\mu}$.

As above, the most interesting issue is whether time allocation can be predicted solely from the pattern of comparative advantage in terms of workers' skills. To determine whether this is the case, again parameterize skills by $\zeta$, and differentiate $q-\mu \zeta t_{\beta}$, taking $\zeta=1$ to be a worker type such that $q-\mu t_{\beta}=0\left(t^{0}\right.$ in Figure 3 is such a type $)$. Then

$$
\begin{aligned}
\frac{\partial}{\partial \zeta}\left(q-\mu \zeta t_{\beta}\right) & =q_{2} t_{\alpha}-\mu t_{\beta} \\
& =q_{2} t_{\alpha}-q \text { at } t^{0} \\
& =q\left(\frac{t_{\alpha}}{q} q_{2}-1\right) \frac{\leq}{>} 0
\end{aligned}
$$

depending on whether $q$ is concave, linear or convex in $t_{\alpha}$. That is $\boldsymbol{\phi}$ is a ray through the origin in Figure 3 if and only if

$$
q\left(1, t_{\alpha}, t_{\beta}\right)=q^{1}\left(1, T_{\beta}\right) t_{\alpha}
$$

In this case, workers having a comparative advantage at task $\alpha$ perform at least as much of that task as do the base group. Assignment by comparative advantage and optimal assignment never conflict. Note that (15) is identical 
(for $\varphi=1$ ) to that which was both necessary and sufficient for the corresponding result in the previous subsection.

When $q(\cdot)$ is not linear in $t_{\alpha}$, the obvious result is: (1) for all worker types performing task $\alpha$ and possessing comparative advantage $\left.\left(t_{\alpha} / t_{\beta}\right)<t_{\alpha} / t_{\beta}<\overline{\left(t_{\alpha} / t_{\beta}\right.}\right)$ in Figure 3, there are other worker types which have a comparative advantage at task $\alpha$ but perform only task $\beta$; and (ii) for all worker types performing only task $\beta$ and possessing comparative advantage satisfying $\left({\left.\underline{t_{\alpha}} / t_{\beta}\right)}\right) t_{\alpha} / t_{\beta}<\left(\overline{t_{\alpha} / t_{\beta}}\right)$, there are workers who have a comparative advantage at task $\beta$ but who perform only task $\alpha$.

4, DISCUSSION

While the above results are derived for an arguably special situation, the same principles hold in a wide range of circumstances. The purpose of this section is to examine a few diverse cases and mention two related points. First, the discussion of market equilibrium was conducted as if the firm in question were the only variety feasible in the economy, or alternatively that only one homogeneous good was produced. w(•) was then determined within the industry. It is possible to carry out the analysis for a small industry taking $w(\cdot)$ as exogenous and allowing variations in product price to limit entry. The assignment results are unchanged by this modification but in general $\ell(t)>0$ only on $\omega \subset \Omega$ (strictly). The set of worker types which will be attracted to the industry can be determined from examination of the set of workers who are indifferent between locations--those $t$ satisfying $\mathrm{px}^{\prime}\left[\mathrm{q}+\left(1-\varphi^{*}\right) \mu \mathrm{t}_{\beta}\right]=\mathrm{w}(\mathrm{t})$ in the diversified case and $\mathrm{px}^{\prime} \cdot \max \left[\mathrm{q}, \mu \mathrm{t}_{\beta}\right]=w(\mathrm{t})$ under specialization. In general, unless $w(\cdot)$ is restricted by more than monotonicity, it does not appear possible to obtain any very useful results on the shape of $w$ (equivalently, the nature of sorting into this industry). Work on a two-sector model wherein $w(\cdot)$ is determined endogenously appears more promising. 
20

A second point is related to the hedonic approach to the determination of wages. Therein, to obtain determinate job-worker matches it is necessary to assume that worker chacteristics cannot be "unbundled". In general, the implicit price of a characteristic then depends on the quantity purchased. The framework studied above is one in which the unbundling assumption is obtained from the underlying structure of production. The asymmetric treatment of tasks $\alpha$ and $\beta$ generates $w(\cdot)$ which is nonlinear in $t_{\alpha}$ and linear in $t_{\beta}$. More generally, when $T_{\beta}$ does not aggregate the $t_{\beta}$ linearly, $w(\cdot)$ will be nonlinear in $t_{\beta}$ as well.

The general collection of ideas in this essay have applicability well beyond the specific instance studied above. To illustrate, consider a twoperson household ( $t^{0}$ and $t^{1}$ ) whose household output $(Q)$ is

$$
Q=q\left[\varphi\left(t^{0}\right), t_{\alpha}^{0}, Y\right]+q\left[\varphi\left(t^{1}\right), t_{\alpha}^{1}, Y\right],
$$

where $\varphi(\cdot)$ is time spent on household activities, $t_{\alpha}$ is ability to perform them and $\mathrm{Y}$ is the value of purchased goods, the public (or at least partially so) good nature of which provides the rationale for family formation. Let $t_{\beta}$ be skills used in the market and $\lambda_{B}$ their rental rate per efficiency unit, in units of the purchased good. Then,

$$
Y=\lambda_{\beta}\left\{\left[1-\varphi\left(t^{0}\right)\right] t_{\beta}^{0}+\left[1-\varphi\left(t^{1}\right)\right] t_{\beta}^{1}\right\}
$$

It follows that, focussing on the diversified case, the condition determining $\varphi *(t)$ is, for $t=t^{0}, t^{1}$,

$$
q_{1}\left[\varphi *(t), t_{\alpha}, Y\right]-\mu t_{\beta}=0
$$

where $\mu \equiv \lambda_{\beta}\left\{q_{3}\left[\varphi *\left(t^{o}\right), t_{\alpha}^{o}, Y\right]+q_{3}\left[\varphi *\left(t^{1}\right), t_{\alpha}^{1}, Y\right]\right\}$

The analysis proceeds exactly as above. 
On a different note, consider a heterogeneous firm model. 12 For simplicity suppose each firm has a fixed labor force comprised of identical (within the firm) workers; heterogeneous entrepreneurs and homogeneous (across firms) workers would do equally well but the analysis is more cumbersome. Suppose task $\beta$ is the production of an intermediate good used in final production, requiring task $\alpha$ and time. The intermediate good may be bought or sold at price H. Aggregate skills within the $i^{\text {th }}$ firm are $t^{i}=\left(t_{\alpha}^{i}, t_{\beta}^{i}\right)$. Given the work force, profit maximization for firm $i$ is equivalent to

$$
\max _{\varphi^{i}, B^{i}} q\left[\varphi^{i}, t_{\alpha}^{i}, B^{i}\right]-\mu I^{i}
$$

where $B^{i}=t_{\beta}^{i}\left(1-\varphi^{i}\right)+I^{i}$ is the quantity $(B)$ of the intermediate good used in production, comprised of own production $t_{\beta}(1-\varphi)$ plus the quantity

purchased, I. Here there are no public good-type gains to cooperation. Again focussing on the diversified case, the necessary conditions for a profit maximum for firm $i$ are

$$
q_{1}-q_{3} t_{B}^{i}=0
$$

and

$$
\mathrm{q}_{3}=\mu \text {. }
$$

Combining these yields

$$
q_{1}\left[\varphi *\left(t^{i}\right), t_{\alpha}^{i}, B^{i}\right]-\mu t_{\beta}^{i}=0 .
$$

Again the analysis proceeds as before.

The primary attribute of these examples which allows them to operate in a manner formally identical to the problem described above is that the efficiency price of $t_{\beta}$ is independent of $t_{\beta}$. While convenient, this is not at all necessary for the kind of results obtained. For example, even in Robinson-Crusoe-type production $q\left[\varphi t_{\alpha},(1-\varphi) t_{\beta}\right]$, where time and skill interact mitiplicatively, time allocation which matches the cross-island pattern of comparative advantage is optimal if and only if $q(\cdot)$ is homothetic. 
Finally, consider an international trade setting in which each of two ( $a$ and $b$ ) countries is endowed with L (normalized to unity) homogeneous workers, each having skills t. Qualities of two goods $\alpha$ and $\beta$, $Q_{\alpha}$ and $Q_{\beta}$ are produced according to $Q_{\alpha}=q_{\alpha}\left(\varphi t_{\alpha}\right)$ and $Q_{\beta}=q_{\beta}\left[(1-\varphi) t_{\beta}\right]$. $\varphi$ is the proportion of the workforce specialized to production of good $\alpha$; the rest produce $\beta$. For output prices $p=\left(p_{\alpha}, p_{\beta}\right)$, the value of output is $v(\varphi, t, p)=p_{\alpha} q_{\alpha}\left(\varphi t_{\alpha}\right)+p_{\beta} q_{\beta}\left[(1-\varphi) t_{\beta}\right]$.

When $q_{\alpha}(\cdot)$ and $q_{\beta}(\cdot)$ are linear, the textbook Ricardian model is obtained. One country will specialize ( $a$ in the production of $\operatorname{good} \alpha, b$ in $\beta$ ) if and only if $t_{\alpha}^{a} / t_{\beta}^{a}>t_{\alpha}^{b} / t_{\beta}^{b}$. Workers are assigned on the basis of comparative advantage. However, if $q_{\alpha}(\cdot)$ is convex and $q_{\beta}(\cdot)$ linear, countries may specialize even if $t_{\alpha}^{a} / t_{\beta}^{a}=t_{\alpha}^{b} / t_{\beta}^{b}$ (Melvin 1969). The same result can occur if country a has a larger labor force or one which has an absolute advantage in the performance of task $\alpha$. Similar arguments imply that countries may or may not specialize and trade on the basis of relative factor endowment as per the Heckscher-Ohlin mode1. 13

\section{SUMMARY AND CONCLUSIONS}

This essay addressed the question of how optimal assignments depend on the endowed traits of economic agents. This question is a very general one which was approached here in the specific setting of assigning workers to tasks within a competitive firm. It was shown, however, that even the restricted structure imposed to analyze the firm was rich enough to be reinterpreted to yield models of time allocation within the family, heterogeneous firms, Robinson Crusoe island economies, and international trade with increasing returns. The most interesting issue in all these cases was whether it is possible to predict the pattern of allocation solely on the basis of 
summary information on the structure of endowed traits. The Principle of Comparative Advantage, when it holds, does exactly that.

The model analyzed in detail--the intra-firm assignment problem-involves determination of how the members of a heterogeneous labor force are assigned to various tasks when the workers of which the labor force is comprised are not perfect substitutes. The particular technology utilized to generate imperfect substitution was one in which final output depended on aggregate individual production, where the latter utilizes workers' time performing one task and the services of a public input. The marginal product of time was assumed nonconstant, and skill in the performance of the task operated as a parameter in the production function. The public input was produced using individual time and ability in the execution of a second task, In the performance of which individual workers are highly substituable. The competitive equilibrium assignment of workers' time was characterized for two cases--pure diversification and pure specialization. In each, cross-worker comparisons of time allocation, individual output and wages were undertaken. The most interesting issue was simply: do workers who have a comparative advantage at some task optimally spend more time performing it? It was shown that except for a razor's edge case, for any given worker there were other workers who both had a comparative advantage at one of the tasks and were optimally allocated less time at the task for which they had a comparative advantage. In general, knowledge of both skills and technology is required to predict the pattern of time allocation.

This result held because the technological structure did not allow all relevant information about absolute skill levels to be summarized by skill ratios. When this is the case, individuals with similar relative skills can have very different assignments because they are very different absolutely. 
Thus the most talented individual may be made company president regard-

less of whether he had a comparative advantage in this activity.

The basic message of all this is simply that only under very special

circumstances are summary measures of endowed traits able to contain all

the information relevant to allocation. 
REFERENCES

Blackorby, C., Primont, D. and Russell, R. R. Duality, Separability.. and Functional Structure: Theory and Economic Applications. New York: American Elsevier, 1978.

Krugman, Paul. "Increasing Returns, Monopolistic Competition and International Trade." Journal of International Economics 9 (November 1979): 469-79.

Markusen, James R. "Factor Movements and Commodity Trade as Complements." Journal of International Economics 14 (May 1983): 341-356. Melvin, James R. "Increasing Returns to Scale as a Determinant of Trade." Canadian Journal of Economics 2 (August 1969): 389-402.

Rosen, S. "Substitution and Division of Labor." Economica 45 (August 1978): 235-50. 
FOOTNOTES

* Thanks for helpful input are due Chris Robinson, Arthur Robson, Sherwin Rosen, Victoria Zinde-Walsh, and the referee.

${ }^{1}$ It is not necessarily assumed that $\mathrm{q}(\cdot)$ is concave. The specific assumptions made concerning $q(\cdot)$ are spelled out below as they are required.

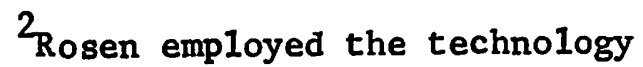

$$
x=x\left(T_{\alpha}, T_{\beta}\right)
$$

where $T_{\beta}$ is as above and

$$
\mathrm{T}_{\alpha}=\int_{\omega} \varphi(\mathrm{t}) \mathrm{t} \alpha \mathrm{dt}
$$

In terms of $(1)-(4)$, this specification can be written $q\left(\varphi, t_{\alpha}, T_{\beta}\right)=\varphi(t) t_{\alpha} f\left(T_{\beta}\right)$, whence $Q=T_{\alpha} f\left(T_{\beta}\right)$. For comparison with what follows, note the linearity of $q(\cdot)$ in $t_{\alpha} \cdot$

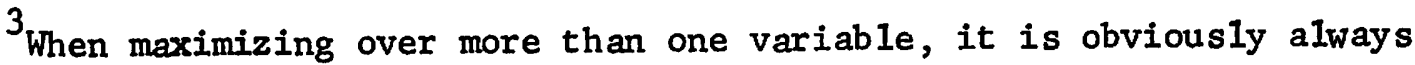
possible to do so sequentially, solving for the optimal value of a subset of variables, conditional on the others. The useful fact here is that under the assumptions made, the first stage problem is to maximize $Q$.

$$
{ }^{4} \text { Failure of } \Psi(\cdot) \text { to be concave in } \ell(\cdot) \text { may occur for two reasons. }
$$

One is that individual outputs are aggregated linearly to obtain $Q$ (i。e. they are perfect substitutes). While this assumption simplifies the analysis considerably, it raises the possibility that it may pay for firms to specialize in hiring workers of a particular type; the pattern of assignment, which is the focus of the analysis, may degenerate. At the 
cost of generating a good deal of algebra, it is possible to obtain results akin to those presented below, when $Q$ is assumed to aggregate individual output in a CES fashion. In the interest of simplicity, the perfect substitution assumption will be maintained.

The second source of nonconcavity of $\Psi(\cdot)$ is the public input assumption. While public inputs provide a nice rationale for the existence of the firm, they create a problem in that some offsetting factor must be imposed to ensure that the firm's optimal employment is finite.

To see just what is required, consider the employment: decision. The marginal return to hiring workers of type $t$ is

$$
\mathrm{px}^{\prime} \cdot\left\{\mathrm{q}+[1-\varphi(\mathrm{t})] \mathrm{t}_{\beta} \mu\right\}
$$

where $\mu \equiv \partial Q / \partial T_{\beta}=\int q_{3} l(t) d t>0$. Hiring one more worker of type $t$ generates individual output of $q$ plus (for $0<\varphi(t)<1$ ) more public input, yielding $[1-\varphi(t)] t_{\beta}(t) \mu$ of output from other workers. The term $p x^{\prime}$ converts marginal aggregate individual output into marginal revenue.

Each additional worker of type $t$ cost $s(t)$. Accordingly, assuming it pays to hire some workers of type $t$, a necessary condition for $h(t)$ to be finite is that marginal returns fall at a rate bounded away from zero. Equivalent ly,

$$
\begin{aligned}
\mathrm{px}^{\prime \prime}\{\mathrm{q} & \left.+[1-\varphi(t)] \mathrm{t}_{\beta} \mu\right\}^{2} \\
& +\mathrm{px}^{\prime}[1-\varphi(t)] \mathrm{t}_{\beta}\left\{2 \mathrm{q}_{3}+[1-\varphi(t)] \mathrm{t}_{\beta} \int_{0}^{1} \mathrm{q}_{33} l(t) \mathrm{d} t\right\}<\epsilon
\end{aligned}
$$

for some $\varepsilon<0$. The arguments familiar from the theory of the firm literature essentially congest the positive externalities responsible for firm 
formation through the use of fixed factors. Here this type of effect arises through $x^{\prime \prime}<0--$ which obtains as a result of the rising ratio of $Q$ to "other factor $s^{\prime \prime}$ in production of final output--and $q_{33}<0$, occurring because of an increasing ratio of private to public inputs in individual production.

$5_{\text {This result holds for } t \in \text { Int } \Omega .} \mathrm{t} \in$ Bndy $\Omega$ requires an obvious minor modification.

${ }^{6}$ It is important to emphasize that the notion of comparative advantage determining the structure of assignment is only useful when it is not tautological, and that it certainly can be made so. For example, in the following subsection a set of skills on which $\varphi(t)$ is constant is identified. Requiring, as seems minimal, that comparative advantage be defined in the space of worker characteristics, it is possible to define comparative advantage by stating that all worker types whose skills lie in the $\varphi$-constant set have identical comparative advantage. Alternatively, given the definition of comparative advantage in terms of $t_{\alpha} / t_{\beta}$, it is in general possible to find some function of $q, \varphi, t_{\alpha}$ and $t_{\beta}$ which is constant as $t_{\alpha}$ and $t_{\beta}$ vary if and only if $t_{\alpha} / t_{\beta}$ is fixed, and then define assignment to be measured by that function. Below it is shown that $\varphi(\cdot)$ is an example of such a function under an appropriate technological restriction. Both these approaches deny comparative advantage any operational content.

$$
\begin{aligned}
& 7_{\text {Using }} \text { (8) } \\
& \qquad \frac{d q}{d t_{\alpha}}=q_{11}^{-1}\left(q_{2} q_{11}-q_{1} q_{12}\right)<0 \Leftrightarrow \frac{q_{2} q_{11}-q_{1} q_{12}}{q_{2}^{2}}>0 \Leftrightarrow \frac{d}{d \varphi}\left(-\frac{q_{1}}{q_{2}}\right)<0 .
\end{aligned}
$$

The last condition is that holding $T_{\beta}$ and $t_{\alpha}$ fixed, an increase in $\varphi$ raises the marginal rate of substitution of $\varphi$ for $t_{\alpha}$, which is equivalent to inferiority of $t_{\alpha}$. 
${ }^{8} t_{\beta}$ varies with $t_{\alpha}$ to hold $\frac{t_{\alpha}}{t_{\beta}}$ fixed in this equation, but $T_{\beta}$ is constant across workers, as is $\varphi$ (by hypothesis).

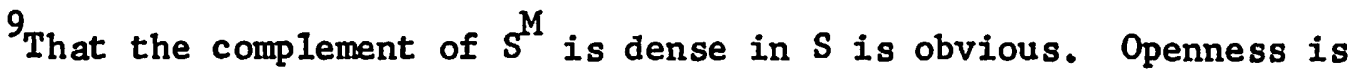
shown by checking that $S^{M}$ is closed (under sup norm) in $S$.

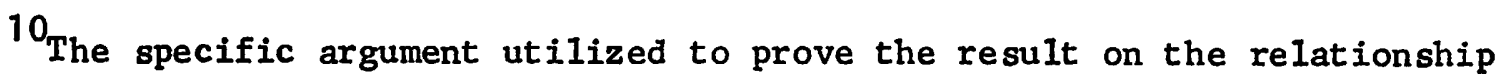
between comparative advantage and optimal assignment hinges on the manner in which those terms are defined. But a more general form of the argument indicates that apart from the tautological methods mentioned in Footnote 6 , construction of measures of comparative advantage and assignment for which comparative advantage determines (or completely summarizes) assignment will be unlikely to meet with success.

The general argument is as follows. Let $C=c(t)$ be a measure of comparative advantage $\left(c\left(t_{\alpha}, t_{\beta}\right) \equiv t_{\alpha} / t_{\beta}\right.$ above) having level sets $c(\bar{c})$ $=\{t \in \Omega / c(t)=\bar{C}\}$. Similarly, let $A=a(t)$ be an index of assignment $(a(t) \equiv \varphi *(t)$ above) with level sets $G(\bar{A})=\{t \in \Omega / a(t)=\bar{A}\} ;$ assume at least one element of each of $\nabla \mathrm{c}$ and $\nabla \mathrm{a}$ is always nonzero. Then comparative advantage completely determines assignment if and only if for all $(\bar{C}, \bar{A})$ such that $C(\bar{C}) \cap G(\bar{A}) \neq\{\phi\}, C(\bar{C})=G(\bar{A})$. Put this way it is immediate that the restriction on $a(t)$ required to achieve correspondence with $a(t)$ is $a(t)=f[c(t)]$, where $f^{\prime}$ has one sign.

In the above analysis $\varphi^{*}(t)$ is a monotonically increasing function of $t_{\alpha} / t_{\beta}$ when $q(a)=q^{1}(\cdot) t_{\alpha}$. More generally, some very strong restriction on $q(\cdot)$ will be required provided, as is minimal for $a(t)$ to make any sense at a11, the dependence of $a(t)$ on $t$ operates at least in part through 
$q(\cdot)$ and/or $\varphi^{*}(t)$. For example, retaining $c(t)=t_{\alpha} / t_{\beta}$, and letting (as is illustrative but not very sensible) $a(t)=q\left[\varphi *(t), t_{\alpha}, T_{\beta}\right]$ implies a differential equation for $q(\cdot)$ having solution $q=\varphi^{a_{1}}{ }_{t_{\alpha}}{ }^{a} h\left(T_{\beta}\right)$, where $1 / 2<a_{1}<1, a_{2}=a_{1} /\left(2 a_{1}-1\right)>1$, and $h^{\prime}>0$.

11 The phrase "at least as much as" appears in the result rather than "strictly more" because $\varphi^{*}$ only takes on two values in the specialized case.

12 This setting can accommodate either free or restricted entry.

13 Markusen (1983) provides a survey of these and related causes of "noncomparative advantage" trade. 

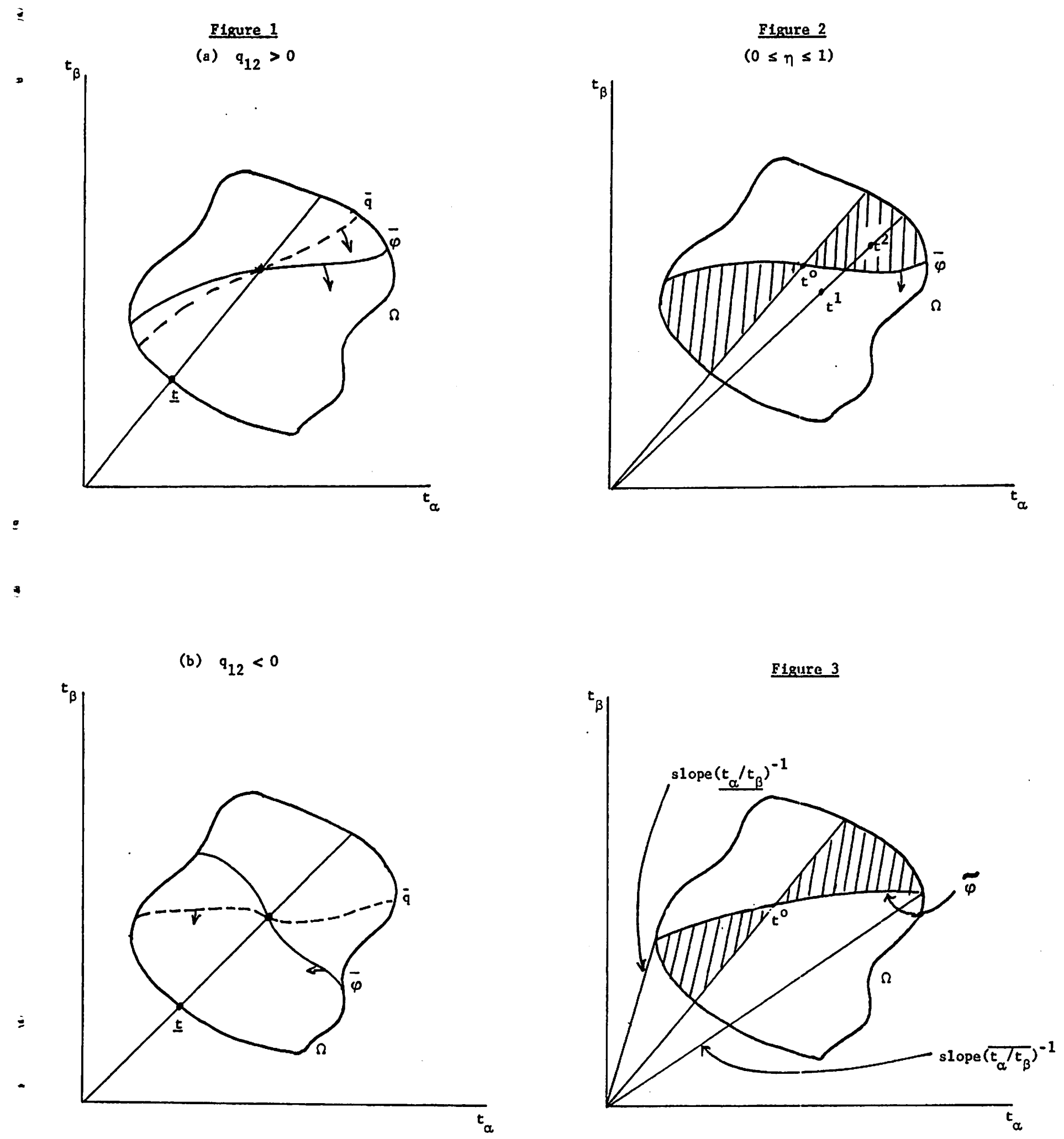